\title{
面向柔性定制的并行不等效客车混装线生产 计划主从联合优化
}

张 炜 ${ }^{1}$ 王少杰 $^{1}$ 甘雅文 ${ }^{1}$ 侯 亮 $^{1}$ 徐昌华 $^{2}$

(1. 厦门大学机电工程系 厦门 361005;

2. 厦门金龙联合汽车工业有限公司 厦门 361023)

\begin{abstract}
摘要: 针对客车制造过程中多条异构混装线之间加工能力、作业时间不等效的特征, 提出面向柔性定制的并行不等效客车混 装线生产计划模型。分析订单分解和投产排序的耦合关联机理; 以产品紧急度、匹配度以及产线负荷为目标, 建立以订单分 解为主、投产排序为从的主从联合优化模型。针对模型特征提出一种结合 Pareto 前沿解的双层交互式遗传算法。为了提高遗 传算法的性能, 引入自适应调整方法对交叉概率和变异概率进行改进, 并采用小生境技术保证种群多样性。利用客车混装线 中的案例对提出的模型进行了验证, 并与多阶段遗传算法以及企业的实际方案进行了比较。所提出的使用双层交互式遗传算 法的模型可以真实地代表企业的实际情况，并最大限度地提高混装线的效率。
\end{abstract}

关键词: 订单分解; 投产排序; 柔性定制; 主从联合优化; 不等效

中图分类号: TH165; TP301

\section{Flexible Customization Oriented Leader-follower Joint Optimization of Production Planning for Parallel and Non-equivalence Bus Mixed-model Assembly Lines}

\author{
ZHANG Wei ${ }^{1}$ WANG Shaojie ${ }^{1}$ GAN Yawen ${ }^{1}$ HOU Liang $^{1}$ XU Changhua ${ }^{2}$ \\ (1. Department of Mechanical and Electrical Engineering, Xiamen University, Xiamen 361005; \\ 2. Xiamen KingLong United Auto Industry Co., Ltd., Xiamen 361023)
}

\begin{abstract}
Due to the non-equivalent processing capacity and working time among multiple heterogeneous mixed-model assembly lines (MMALS) in the bus manufacturing process, a parallel non-equivalent production planning model for flexible customization is proposed. By analyzing the interactions between the order decomposition and production sequencing, a leader-follower joint optimization model that takes the order decomposition as the leader and production sequencing as the follower is proposed, in order to optimize the product urgency, matching degree and production line load. A bi-level interactive genetic algorithm with Pareto front-line solution is developed to solve the model. To improve the performance of the genetic algorithm, an adaptive adjustment method is introduced to guarantee the crossover and mutation probabilities and niche technology to ensure the diversity of population. The proposed model is verified using cases in MMALS of buses and compared with other two methods, namely, the multistage genetic algorithm and the actual scheme of the enterprise. The proposed model that uses the bi-level interactive genetic algorithm can represent the actual situation of the enterprise and maximize the efficiency of MMALS.
\end{abstract}

Key words: order decomposition; production sequencing; flexible customization; leader-follower joint optimization; non-equivalence

\section{0 前言}

定制化时代, 产品品种、功能需求快速增长,

* 国家自然科学基金资助项目(51975495)。20200401 收到初稿, 20200703 收到修改稿
订单数量、交期呈现高度动态性 ${ }^{[1]}$ ，以 “顾客为中 心”的柔性定制日趋重要 ${ }^{[2-3]}$, 使传统客车制造企业 面临诸多挑战。由于需要生产不同规格、类型的客 车产品, 基于具有相似功能的多条异构混装线的订 单制造和柔性定制成为企业首选。但目前多数研究 
只以最小化产品作业任务的完成时间或拖期为优化 目标 ${ }^{[4-6]}$, 很少考虑订单分解和投产排序之间的耦合 关系，也没分析多条异构混装线的不等效特征，使 得模型和预期结果与企业实际需求有较大偏差。

柔性定制背景下考虑并行不等效特征的订单分 解和投产排序, 需要解决以下两个的问题: 首先, 在考虑不同混装线加工能力、作业时间上存在不等 效特征的基础上, 对当前订单进行分解实现不同混 装线生产任务的合理分配; 其次, 基于不同混装线 各个工作站之间的负荷均衡, 将分配至各条混装线 的产品进行投产顺序优化。以上工作是保证企业混 装线顺利运行的关键因素。

生产计划的首要任务是订单分解, 订单分解问 题是指如何将企业当前订单合理地分解至各个混装 线 ${ }^{[7]}$ 。一般是采取基于订单顺序和产品交期统筹考 虑的方法 ${ }^{[8]}$ 。这种方法能够将产品作业时间和设备 产能进行协同, 在满足生产计划可行性的同时兼顾 了设备负荷的均衡性 ${ }^{[9]}$ 。基于上述原则, 郑华林等 ${ }^{[10]}$ 提出了一种将粗细排产相结合的联合调度优化方 法。WUNSCH 等 ${ }^{[7]}$ 基于实际制造系统, 研究了一个 多级的订单分解机制, 很大程度上增强了企业排产 的灵活性。然而, 上述方法基本上只考虑如何将订 单分解至各条混装线, 至于各条混装线的具体投产 顺序则没有深入分析。

订单分解的结果是确定各条混装线的投产顺序 前提条件。产品投产排序问题, 是指通过合理地将 相似产品按照一定顺序在同一混装线上加工, 以快 速响应的个性化需求 ${ }^{[11-12]}$ 。范林胜等 ${ }^{[13]}$ 以均衡化生 产为目标, 构建焊装车间作业计划优化模型。以工 作站负荷均衡为目标, 采用分支定界法对总装车间 投产排序的问题进行研究 ${ }^{[14]}$ 。考虑车型所涉及的不 同颜色，对涂装车间车辆的投产顺序进行规划 ${ }^{[15]}$ 。

许多研究人员采用先订单分解后投产排序的 “分阶段递阶” [16]优化的方法进行优化求解。这种 方法在大批量、少品种的生产线中较为常见。随着 产品生产方式转为多品种、小批量之后, 这种方法 由于忽略了产能、设备约束, 常出现预先指定的订 单分解任务无法合理安排而导致交期延误的情况。

针对上述问题, 研究人员综合考虑不同层次的 约束条件, 以期构建整体模型。例如, 王艳红等 ${ }^{[17]}$ 在处理生产计划和调度问题时, 利用动态约束平衡 的混合算法进行协同优化。王静等 ${ }^{[8]}$ 引入订单拆分 数和订单最小分解率变量, 在生产时间和产能约束 下构建生产计划的优化模型。它虽然避免层次优化 方法得到不可行解或收玫于局部解, 但计算量大,
求解效率低。另外, 模型在归一过程中往往掺杂了 许多主观的因素, 使得优化结果具有一定的倾向性。

在工程领域中, 许多工程设计决策问题都具有 多主体、多目标、多约束的特点, 涉及到满足整 体问题目标所包含的各种权衡和约束。考虑个性 化需求的并行不等效混装线生产计划问题也具有 上述特点。现有一体化方法没有把握所研究问题 的多主体、多目标和多约束的本质特点, 将不同 领域的目标放在同一范围内、并一次性决定所有 的设计变量。

考虑到订单分解和投产排序所具有的多决策准 则特性, 且某些决策方案包含多个相互竞争等问题, 必须相互妥协才能达到均衡最优。因此, 这种多个 竞争优化问题共同导致了一个联合优化问题, 它是 复杂工程系统中的常见问题, 涉及多个子系统的多 种耦合, 通常需要子系统的协同优化。

事实上, 混装线生产计划问题除了多主体和多 目标的特点外, 还存在问题的层次性。一般而言, 个性化需求背景下的企业会更多地考虑订单的紧急 性, 因此紧急性在整个联合优化系统中占据主导地 位, 而混装线线投产排序占据相对次要地位。

上述问题属性的分析, 对于获得混线生产计划 最佳性能非常重要。主从联合决策优化方法, 最适 合求解此类问题, 尤其是决策者主导整个决策过程 的情形。在主从联合优化模型中, 订单分解和投产 排序问题都有独立的目标函数和约束条件。两个问 题既相互关联又具有相对的独立性。处于从属地位 的投产排序必须服从强势地位的订单分解的设计决 策, 优化过程是反复迭代的过程。如图 1 所示, 一 方面, 上层订单分解主模型的优化结果作为投产排 序的输入，其结果直接决定了从者投产排序所导致 的混装线效率的好坏; 另一方面, 下层投产排序从 模型的优化方案反馈给订单分解, 对订单分解主模 型优化结果的优劣进行合理性评估。两者各自的目 标构成了生产计划的总体目标, 需要循环迭代优化 才能得到综合性能最优的结果。上述优化过程是订 单分解和投产排序之间的整体和局部的主从关联优 化。这种关联因素之间并不是并列的, 而是分层次 的主从关联关系。

柔性定制背景下进行订单分解和投产排序问题 优化时, 还需要考虑各条混装线之间的不等效问题。 当前许多研究为了简化模型, 往往只针对某一条生 产线的问题进行优化, 没有考虑不同混装线对产品 投产排序的协同关系。由于客车行业制造系统存在 多条并行混装线且不等效的特征, 导致模型与企业 
实际需求之间存在差异。

客车企业的异构混装线存在以下特点：首先， 由多条包含多道工序的并行混装线组成, 该类混装 线订单分解和投产排序问题是混装线排序问题和多 条混装线之间协同问题的集成 ${ }^{[18]}$ 。其次, 并行混装 线及其各个工作站功能相似, 但每条混装线存在着 人员、设备、空间等方面的差异 ${ }^{[19-20]}$ 。当面对不同 的客车产品时, 不同混装线会体现出加工能力、作 业时间等不一致的不等效特性。
国内外相关领域学者对多生产线协同问题 进行了大量的研究 ${ }^{[21-22]}$, 但这些方法忽略了实际 生产过程中各条混装线各个工作站存在不等效 差异这一重要特点。为了有效表征不同混装线加 工不同产品时所体现出来的不等效特性, 采用了 匹配度目标函数 $F_{2}$ 来量化这种差异。根据不同产 品和不同混装线之间匹配度值, 得到订单分解后 各条混装线和不同产品的匹配度综合值，具体的 数学模型见式(9)。

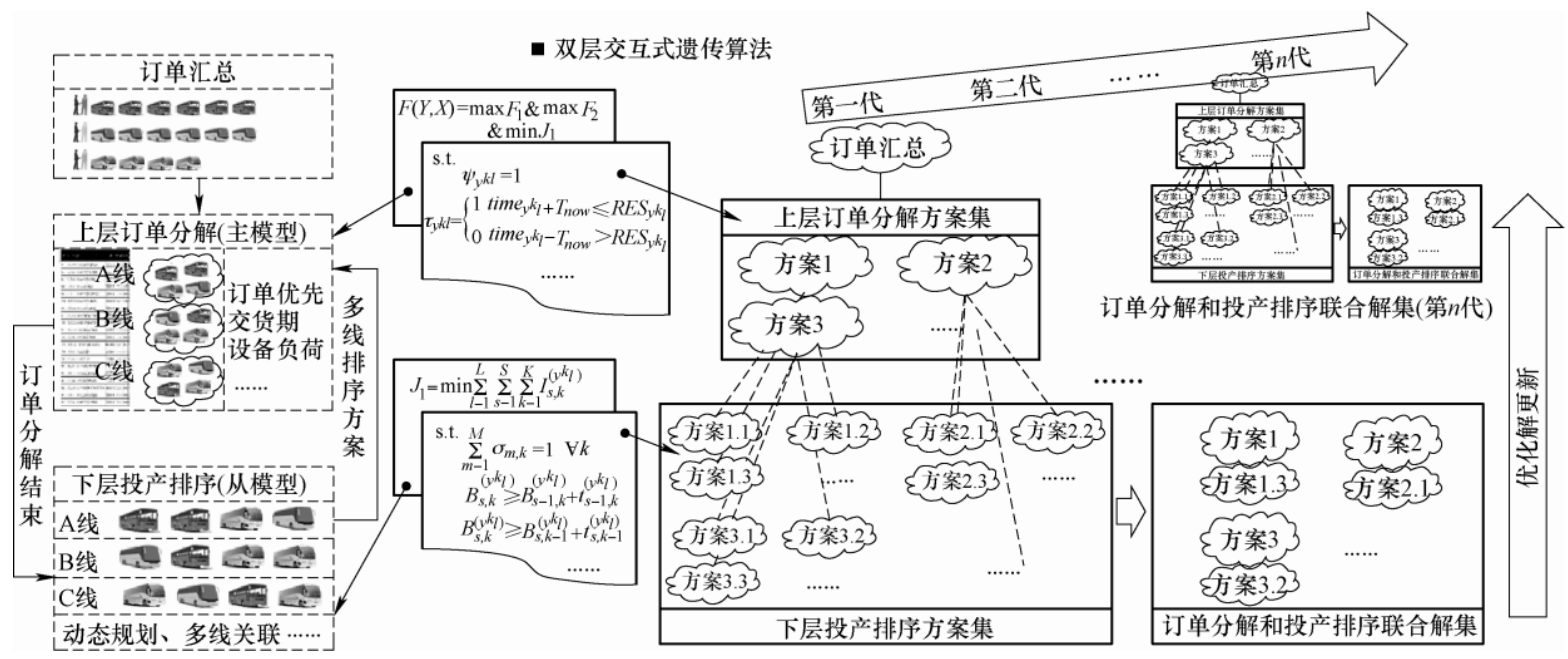

图 1 基于双层交互式遗传算法的生产线规划

综上所述，鉴于订单分解和投产排序之间的耦 合关系, 以及客车行业制造系统存在多条并行混装 线且不等效的特征, 建立一个考虑混装线并行不等 效特征的生产计划主从关联优化模型。相对于传统 的多目标优化模型, 本文模型保留了集成优化中所 忽略的主从关联关系, 也强调了多阶段优化所忽略 的循环迭代关系。同时, 模型还考虑了企业实际存 在的各条生产线不等效的特征。

本研究不同于传统混装线生产计划, 它是混装线 生产计划的延伸。其原因如下: (1) 从单个混装线延 伸到多个混装线; (2) 并行混装线从简单相同的生产 线延伸到考虑产品与不同混装线之间匹配度的问题;

(3) 考虑订单分解和投产顺序的主从关联关系。

针对模型的各优化目标之间存在相互约束甚至 相互冲突的特征, 提出了一种结合 Pareto 前沿解的 双层交互式遗传算法进行求解。将该方法应用到某 企业并行不等效混装线上, 与企业实际的订单分解 和投产排序方案, 以及采用多阶段遗传算法的优化 结果进行对比。对比的结果显示, 本文提出的主从 关联优化模型及相应算法能更有效地解决订单分解 和投产排序关联优化问题, 使企业并行混装线的效 率得以提升。

\section{1 并行不等效混装线生产计划模型}

某制造企业的 $C$ 个客户向企业提交生产 $N$ 种 产品的 $H$ 个订单，这些订单会被分配到企业内一组 不完全相同的混装线。每条混装线都有对于不同产 品而言不等效的串联起来的多个工作站, 其处理速 度不同。一组不同的产品, 它们需要通过一组的作 业程序(混装线)以完成某种产品的加工。

订单在计划周期内有 “生产或者延迟” 两种处 理方式, 订单信息包含产品需求量和产品交货期等。 模型需要决策: (1) 各个订单分解给哪些混装线, 数 量是多少; (2) 各条混装线的具体投产顺序如何安 排, 使产品能够在交期内完成。模型中的集合和参 数如表 1 所示, 决策变量如表 2 所示。

混装线订单分解和投产排序的优化问题实际上 是一个主从关联优化问题，基于此构建决策框架体 系。该框架体系中的主模型是订单分解, 从模型是 投产排序，本文以 $\boldsymbol{X}$ 和 $\boldsymbol{Y}$ 分别代表主从设计变量。 基于决策框架体系，决策人员通过优化目标 $F(\boldsymbol{X}, \boldsymbol{Y})$ 和主模型的约束对设计变量 $\boldsymbol{X}$ 进行决策优 化。上述优化结果和相应的决策变量 $\boldsymbol{X}$ 将被传递到 
下层从者。从者基于上述前提及自身的优化目标 $f(\boldsymbol{X}, \boldsymbol{Y})$ 以及约束对设计变量 $\boldsymbol{Y}$ 进行决策。用一个 双层规划模型来表达主从关联优化问题, 其通用形 式如下 ${ }^{[23]}$

$$
\left\{\begin{array}{lll}
\max _{\boldsymbol{X}} F(\boldsymbol{X}, \boldsymbol{Y}) & \text { s.t. } & \left\{\begin{array}{l}
G(\boldsymbol{X}) \geqslant 0 \\
H(\boldsymbol{X})=0
\end{array}\right. \\
\min _{\boldsymbol{X}} f(\boldsymbol{X}, \boldsymbol{Y}) & \text { s.t. } & \left\{\begin{array}{l}
g(\boldsymbol{X}) \geqslant 0 \\
h(\boldsymbol{X})=0
\end{array}\right.
\end{array}\right.
$$

表 1 集合和参数表

\begin{tabular}{|c|c|c|c|}
\hline 符号 & 注释 & 符号 & 注释 \\
\hline$F_{1}$ & 订单分解的紧急度模型 & $F_{2}$ & 订单分解的匹配度模型 \\
\hline$i, n$ & 总订单的产品类型 $(i, n=1,2, \cdots, N)$ & $l$ & 生产线类型 $(l=1,2, \cdots, L)$ \\
\hline$x_{n, l}$ & 分解至产线 $l$ 上的产品 $n$ 的总量 & $H$ & 订单产品总数量 \\
\hline $\mathrm{Num}_{n}$ & 不同类型产品的订单数量 & time $_{n}$ & 不同类型产品的交期时间 \\
\hline$M A_{n, l}$ & 不同类型产品与不同生产线的匹配度 & $T_{p}$ & 经验生产周期 \\
\hline$E_{n}$ & 交期时间在产品 $n$ 前的车辆总数 & $\varepsilon$ & 计划排程的经验预留时间 \\
\hline$R_{l}$ & 分解至生产线 $l$ 上的总产品 & $T_{\text {now }}$ & 当前计划分解时间 \\
\hline$J_{1}$ & 某条焊装线生产排序的工作负荷模型 & $R E S_{n}$ & 动态预留时间 \\
\hline$m$ & 分解至生产线的车辆类型 $(m=1,2, \cdots, M)$ & $\eta$ & 临界订单加权因子 \\
\hline$s$ & 混合生产线上的工作站 $(s=1,2, \cdots, S)$ & $P R_{l}$ & 生产线生产能力 \\
\hline$k$ & 排序产品的具体位置 $(k=1,2, \cdots, K)$ & $J_{2}$ & 某条生产线的产品切换次数模型 \\
\hline$t_{s}^{(m)}$ & $m$ 型产品在工作站 $s$ 上的作业时间 & $y^{k_{l}}$ & 生产线 $l$ 在排序位置 $k$ 上的产品类型 \\
\hline simi & 个体相似度 & $D_{m}$ & 每种类型产品的生产量 \\
\hline$Q_{s, k}^{\left(y^{k_{4}}\right)}$ & $\begin{array}{c}\text { 生产线 } l \text { 上投产顺序为 } k \text { 的产品, 在工作站 } s \text { 结束加工的 } \\
\text { 时间 }\end{array}$ & $t_{s, k}^{\left(y^{k_{l}}\right)}$ & $\begin{array}{c}\text { 生产线 } l \text { 上投产顺序为 } k \text { 的产品, 在工作站 } s \text { 上的加工 } \\
\text { 时间 }\end{array}$ \\
\hline$B_{s, k}^{\left(y^{k q}\right)}$ & $\begin{array}{c}\text { 生产线 } l \text { 上投产顺序为 } k \text { 的产品, 在工作站 } s \text { 上开始加工 } \\
\text { 的时间 }\end{array}$ & $I_{s, k}^{\left(y^{k}\right)}$ & $\begin{array}{c}\text { 生产线 } l \text { 上投产顺序为 } k \text { 的产品, 在工作站 } s \text { 时的空闲 } \\
\text { 堵塞时间 }\end{array}$ \\
\hline
\end{tabular}

表 2 决策变量表

\begin{tabular}{|c|c|c|c|}
\hline 符号 & 注释 & 符号 & 注释 \\
\hline$\psi_{n, i}$ & 产品 $i$ 交期时间是否在产品 $n$ 前, 为 1 , 否则为 0 & $\varpi_{n, l}$ & 产品 $n$ 分解至生产线 $l$, 为 1 , 否则为 0 \\
\hline$\tau_{n}$ & 产品 $n$ 为紧急订单, 为 1 , 否则为 0 & $\sigma_{m, k}$ & 在 $k$ 位置的产品为 $m$ 型, 为 1 , 否则为 0 \\
\hline$\delta_{\text {pop-ctg }}^{k}$ & $\begin{array}{c}\text { 在 } k \text { 位置的 pop 个体和类 } c t g \text { 个体的产品类型一 } \\
\text { 样, 为 } 1 \text {, 否则为 } 0\end{array}$ & $\theta_{y^{k_{l}}, y^{(k+1) l}}$ & $\begin{array}{c}\text { 生产线 } l \text { 在 } k \text { 位置的 } y^{k_{l}} \text { 型产品和在 } k+1 \text { 位置的 } \\
y^{(k+1)} \text { 型产品一致, 为 } 1 \text {, 否则为 } 0\end{array}$ \\
\hline
\end{tabular}

上述优化模型能够体现了订单分解和投产排序 之间的耦合关系。基于上述一般模型，建立生产计 划问题的主从关联优化模型, 并对模型的上下层定 义及两者的主从关联关系进行研究。

\section{1 上层订单分解优化模型}

已知企业各条混装线 $l$ 及其生产能力 $P R_{l}$, 当前 排产周期内总订单的产品类型 $n$ 及其订单量 $N u m_{n}$ 和交期 time $_{n}$, 各产品与各混装线之间的匹配度 $M A_{n, l}$, 需要对当前排产周期内总订单的产品进行订 单分解。首先, 基于客户的需求考虑产品紧急度; 其次, 考虑到不同混装线的不等效特征, 以产品和 混装线之间的匹配度为目标。
订单分解决策变量的是生产线分配到的订单状 况。 $h$ 为当前总订单的产品总数量 $H$ 下第 $h$ 款产品, $(h=1,2, \cdots, H)$ 。生产线 $l(l=1,2, \cdots, L)$ 的产品选择向 量 $X=\left\{x_{1,1}, \cdots, x_{H, L}\right\}$ 。其中， $x_{h, l}$ 是一个 $0-1$ 变量, $x_{h, l}=1$ 表示将产品 $h$ 分解至生产线 $l$ 上生产; $x_{h, l}=0$ 表示将产品 $h$ 不分解至生产线 $l$ 上生产。

\section{1 紧急度}

紧急度目标函数建立的目的是判断当前排产 周期内各产品的紧急程度。为此, 首先对各款产 品的交期时间进行排序。交期时间在产品 $n$ 前的 车辆总数 


$$
E_{n}=\sum_{i}^{N} \psi_{n, i} \cdot \operatorname{Num}_{i} \quad \forall \psi_{n, i}
$$

当 time $_{i} \leqslant$ time $_{n}$ 时, $\psi_{n, i}$ 赋值为 $1 ;$ time $_{i}<$ time $_{n}$ 时, $\psi_{n, i}$ 赋值为 0 。基于产品数量与交期时间得到订单中 不同车型的动态预留时间是

$$
R E S_{n}=\left[\frac{\sum_{i}^{N} N u m_{i} \cdot \psi_{n, i}}{\sum_{l}^{L} P R_{l} \cdot l}\right]+1+T_{p}+\varepsilon \quad \forall \psi_{n, i}
$$

基于订单紧急度的分析可以对当前订单进行合 理的分配。通过不同类型车辆的交期时间 time $_{n}$ 与该 车型所需的动态预留时间 $R E S_{n}$ 之间的对比来判断 该车型的紧急程度。为此, 引入布尔变量 $\tau_{n}$ 用来表 示车型 $n$ 在当前订单中应该处的分解状态。若 time $_{n}-T_{\text {now }} \leqslant R E S_{n}$, 则表示车型 $n$ 为紧急订单; 取 $\tau_{n}=1$ 以提高车型 $n$ 的紧急度, 使其在投产时被优先 考虑。若 $t i m e_{n}-T_{\text {now }}<R E S_{n}$, 则认为订单可以被正 常分解, 取 $\tau_{n}=0$ 。车型 $n$ 的紧急度模型如下

$$
F_{n}=\left(\tau_{n} \cdot \eta+\left|\tau_{n}-1\right|\right) \cdot \frac{R E S_{n}}{\text { time }_{n}-T_{\text {now }}}
$$

序号 $k$ 为分解至某生产线 $l$ 上的排序位置, 用 $k_{l}$ 表示。某条生产线 $l$ 在生产排序 $k$ 位置上的具体产 品类型为 $y^{k_{l}}$ 。企业订单分解时的整体紧急度函数为

$$
\begin{aligned}
& F_{1}=\max \sum_{l=1}^{L} \sum_{h=1}^{H} \sum_{k=1}^{K}\left(\tau_{y^{k_{l}}} \cdot \eta+\left|\tau_{y^{k_{l}}}-1\right|\right) \times \\
& x_{h, l} \cdot \frac{\left[\frac{\sum_{i}^{N} N_{l} m_{i} \cdot \psi_{y^{k_{l}, i}}}{\sum_{l}^{L} P R_{l} \cdot l}\right]+1+T_{p}+\varepsilon}{\text { time }_{y^{k_{l}}}-T_{\text {now }}} \\
& \text { s.t } \quad \sum_{l=1}^{L} \sum_{k=1}^{K} x_{y^{h^{h}, l}} \leqslant \sum_{l=1}^{L} \sum_{n=1}^{N} N u m_{n} \quad \forall n \\
& \psi_{y^{k_{l}, l}}=1 \\
& \tau_{y^{k_{l}}}=\left\{\begin{array}{l}
1 \text { time }_{y^{k_{l}}}-T_{\text {now }} \leqslant R E S_{y^{k_{l}}} \\
0 \text { time }_{y^{k_{l}}}-T_{\text {now }}>\operatorname{RES} S_{y^{k_{l}}}
\end{array}\right.
\end{aligned}
$$

约束(6)保证总的生产订单量不大于计划订单量; 约 束(7)得到交期时间不晚于待分解订单的全部订单 量; 约束(8)为临界订单和正常订单的判断条件。

\subsection{2 匹配度}

匹配度目标函数的建立是基于不同混装线加工 相同产品存在不等效特征而考虑的。客车并行混装 线有着复杂工艺流程的相似设备。传统生产计划方 案将同一订单的产品分配到同一条混装线上生产。
柔性定制时代的来临，订单的数量和交期呈现较大 的差异性，按照产品和混装线匹配的方式一方面造 成单条混装线任务繁重且难以满足客户订单需求, 另一方面又造成其他混装线闲置。

因此，基于柔性定制背景下不同产品的生产计 划和批量、交期差异，考虑不同混装线总体负荷均 衡, 将同一订单拆分到具有相同/相似属性的不同混 装线。这种分配过程不是随意的, 还要充分考虑不 同混装线各种功能相同/相似的工作站装置对处理 同类型零部件所需加工作业时间的差异性、装配成 本的不同等不等效特征。

不同混装线存在着人员作业习性、设备布局等 方面的差异，使其在加工相同产品时所耗费的时间 不同。例如, 用于焊装小型客车骨架的混装线, 当 投入大型客车骨架产品时, 会导致各个工作站布局 更为紧凑, 不利于焊接作业的顺利完成。因此, 产 品只有在其适配的装配线生产，才能最大发挥混装 线的制造能力。

基于上述因素，构建考虑产品与混装线之间匹 配度的参数指标 $M A_{n, l}$, 即混装线 $l$ 与产品 $n$ 的匹配 度。匹配度值是一个包含了作业时间、加工工艺、 产品尺寸和结构等众多因素的综合经验值, 取值范 围为 $[0.8,1]$ 。匹配度值越高, 说明该混装线越适合 加工该产品。

假设有 $R_{l}$ 个产品分解至混装线 $l$ 上, $y^{k_{l}}$ 为混装 线 $l$ 在投产排序 $k$ 位置上的具体产品类型。针对产品 的分解情况，建立订单分解的匹配度函数 $F_{2}$ 为

$$
\begin{gathered}
F_{2}=\max \sum_{l=1}^{L} \sum_{h=1}^{H} \sum_{k=1}^{K} x_{h, l} \cdot M A_{y^{k, l}, l} \\
\text { s.t. } \quad \sum_{l=1}^{L} \sum_{n=1}^{N} x_{h, l}=\sum_{l=1}^{L} R_{l}
\end{gathered}
$$

式中, 约束(10)确保分解至生产线 $l$ 的产品全部投入 生产排序中。MA $A_{y^{k, l},}$ 指的是混装线 $l$ 于其排序 $k$ 位置 上的产品的匹配度。

上层的目标函数除了紧急度和匹配度之外, 还 综合了投产排序所产生的负荷均衡问题。因此, 上 层模型实际上是综合了紧急度 $F_{1}$ 、匹配度 $F_{2}$ 、负荷 均衡 $J_{1}$ 这三个目标的多目标优化模型。由于这三个 目标之间通过决策变量相互制约, 且各目标所考虑 的维度、意义不同, 很难客观评价多目标解的优劣 性。因此, 在寻优时通过 Pareto 前沿解的方式进行 优化求解, 优化求解所得的是一个最优解集合。

\section{2 下层投产排序优化模型}

虽然任何的投产排序方案都可以用于实际生 
产, 但合理的、科学的生产计划, 能够提高混装线 上产品的加工效率 ${ }^{[24-25]}$ 。多个相同产品连续投入到 同一混装线会使某些站点出现持续负荷过载。高、 低负荷产品交替通过相同站点可以减少这种负荷不 均衡性。这种均衡化的投产方式有效解决工位负荷 过载的问题 ${ }^{[26]}$ 。本节针对实际生产过程中混装线负 荷过载问题，建立以最小化工作负荷为目标的投产 排序模型。

假设某一混装线需装配 $M$ 种产品, 产品品种为 $m(m=1,2, \cdots, M)$, 各种产品的产量是 $D_{m}$ 。则混装 线 $l$ 上投产顺序为 $k$ 的产品在站点 $s$ 上的加工时间 为

$$
t_{s, k}^{\left(y^{k_{l}}\right)}=\sum_{m=1}^{M} \sigma_{m, k}^{l} \cdot t_{s}^{(m)}
$$

若生产线 $l$ 上位置 $k$ 的产品不是 $m$ 型, $\sigma_{m, k}^{l}=0$; 若生产线 $l$ 上位置 $k$ 的产品是 $m$ 型, $\sigma_{m, k}^{l}=1$ 。

混装线上的产品必须等待其下一个站点完成现 有装配任务, 才能进入到下一个站点。如果下一个 站点并未完成, 则需要在原站点进行等待。该工作 站的空闲堵塞时间 ${ }^{[15]}$ 为

$$
I_{s, k}^{\left(y^{k_{l}}\right)}=\left|Q_{s-1, k}^{\left(y^{k_{l}}\right)}-Q_{s, k-1}^{\left(y^{k_{l}}\right)}\right|
$$

参照郑永前等 ${ }^{[27]}$ 的等待时间和堵塞时间方程 式, 构建基于工位空闲和产品堵塞时间的工作负荷 函数

$$
\begin{gathered}
J_{1}=\min \sum_{l=1}^{L} \sum_{s=1}^{S} \sum_{k=1}^{K} I_{s, k}^{\left(y^{k_{l}}\right)} \\
\text { s.t. } \sum_{m=1}^{M} \sigma_{m, k}=1 \quad \forall k \\
\sum_{k=1}^{K} \sum_{m=1}^{M} \sigma_{m, k}=K \quad \forall m \quad \sigma_{m, k} \in\{0,1\} \\
B_{s, k}^{\left(y^{k l}\right)} \geqslant B_{-11}^{6^{k_{l}}}, \frac{\hbar}{k} \underline{t}_{s 1}^{y^{k^{l}}} \\
B_{s, k}^{\left(y^{\left.k^{k}\right)}\right.} \geqslant B_{s, k-1}^{\left(y^{k_{l}}\right)}+t_{s, k-1}^{\left(y^{k_{l}}\right)}
\end{gathered}
$$

约束(14)确保在生产计划周期内, 不同站点上 分别只有一个产品在加工。约束(15)确保在生产计 划周期内, 投入到混装线的产品总数等于计划周期 内产量。约束(16)确保第 $k$ 个排序的产品, 在结束站 点 $s-1$ 的作业后, 才可以进入站点 $s$ 进行新的作业。 约束(17)确保站点 $s$ 须先装配第 $k-1$ 个排序的之 后, 才能装配第 $k$ 个的产品。

\section{3 主从联合优化模型}

基于上下层之间的关系，上层目标函数是包含 了 $F_{1}, F_{2}, J_{1}$ 不同维度的综合模型, 为此构建 Pareto
数学模型如式(18)所示。下层以最小化工作负荷为 目标函数, 如式(20)所示。建立订单分解和投产排 序的主从关联优化模型如下

$$
\begin{gathered}
F(X, Y)=\max F_{1} \& \max \quad F_{2} \& \min J_{1} \\
\text { s.t. } \text { 式 }(6) \sim(8),(10) \\
f(X, Y)=J_{1}=\min \sum_{l=1}^{L} \sum_{s=1}^{S} \sum_{k=1}^{K} I_{s, k}^{\left(y^{k^{l}}\right)} \\
\text { s.t. 式 }(14) \sim(17)
\end{gathered}
$$

\section{2 双层交互式遗传算法}

模型(18)的求解方法依据其模型规模和构成函 数的复杂度决定, 其构成函数包含了下层的目标函 数 $J_{1}$ 。因此, 采用双层规划方法进行寻优。相对于 传统的采用微积分的优化方法而言, 遗传算法在寻 优过程中参考自然界的自然选择和遗传机制的随机 化搜索方法, 更适合求解此类多参数、多变量、多 目组合优化问题。

本文针对主从关联优化问题的特征, 对遗传算 法进行改进，构造适应模型问题特性的双层交互式 遗传算法。根据模型上下层之间交互反馈的关系， 首先对总订单进行分析, 根据订单的总量、紧急程 度等因素获得需要分解的订单。以此为基础, 通过 遗传算法获取订单分解方案; 下层则根据订单分解 方案进行生产排序优化, 并将结果传递给上层。上 层根据订单分解和生产排序的结果进行协调寻优, 并将新的结果传递给下层。如此形成一个闭环优化, 并逐渐收玫, 得到优化解集。最后, 对 Pareto 前沿 解集, 利用量纲归一化的方法决策出最后结果。

由于遗传算法容易出现 “早熟”、收敛速度慢等 缺点, 本文在针对主从关联优化模型设计了双层交 互式遗传算法的基础上，在交叉概率和变异概率的 值的设定方面采用自适应调整的方法进行改进。在 种群选择分类方面引入了小生境技术, 将所有个体 分成多个种群。采用自适应小生境双层交互式遗传 算法, 能够保持种群的多样性, 并使算法能够较快 实现收敛。自适应方法基于上层目标和下层目标的 适应度函数值, 对当前解的质量进行有效甄别。由 于引入了调整系数, 在进化的过程中既考虑了进化 后代的集中性, 又考虑了收玫方向, 使得算法能够 较快地向全局最优解收敛。

\section{1 基于小生境技术的选择操作}

小生境指的是在特殊环境下的一种组织功能。 在生物界, 往往特征、性状类似的物种聚集会起来, 
通过交配进行繁衍。这种技术应用到计算科学领域 时, 将特征相似的数据进行聚类, 每类中适应度值 较好的个体将予以保留。对每一类中的剩余个体则 进行交叉和变异, 以产生新一代个体子群 ${ }^{[15]}$ 。具体 操作步骤如下。

(1) 个体聚类。首先, 通过种群中当前个体的 历史最优位置集合得到独立性最好的个体, 即中心 个体。其次, 分析计算剩余个体与上述中心个体之 间的相似度。按照所得相似度的最大值将剩余个体 归类至该中心个体。如果出现某剩余个体的两个相 似度值相等的情况, 则随机归类给其中任何一类。 相似度公式如下

$$
\operatorname{simi}=\sum_{k=1}^{K} \delta_{p o p-c t g}^{k}
$$

式中, pop 个体与类 $c t g$ 个体在第 $k$ 位置的值一致, 则 $\delta_{p o p-c t g}^{k}=1$; 如果 pop 个体与类 $c t g$ 个体在第 $k$ 位 置的值不一致, 则 $\delta_{p o p-c t g}^{k}=0$ 。

(2) 同类个体分层。通过遍历的方式对 $P$ 个个体 的相互支配关系进行计数统计, 实现对整个种群分层。 计算种群 $P$ 中的每个个体 $p$ 的两个参数 $n_{p}$ 和 $S_{p}$ 。其 中, $n_{p}$ 为种群内能够支配 $p$ 的个体数量, $S_{p}$ 则是种 群内被 $p$ 支配的个体集合。算法的具体步骤如下。

1) 搜索种群内 $n_{p}=0$ 的个体, 并将其储存在集 合 $F_{l}$ 内。

2) 对当前个体集合 $F_{l}$ 内的任意一个体 $i$, 其能够 支配的个体集合是 $S_{i}$, 遍历 $S_{i}$ 中的所有个体 $l$, 并让 $n_{l}=n_{l}-1$, 如果 $n_{l}=0$ 则将个体 $l$ 储存在集合 $H$ 中。

3) 将 $F_{l}$ 中的个体标识为第一个非支配层的个 体, 设 $H$ 为当前集合, 重复上述过程, 直至整个种 群实现分级。

（3）个体择优。通过构造个体的储存库来存储 和决定每一代中的哪些个体将成为非支配解的成 员, 并指导其他个体的更新。在外部储存库的更新 阶段, 将所有非支配解输入外部储存库, 然后消除 彼此靠近的个体(图 2)。

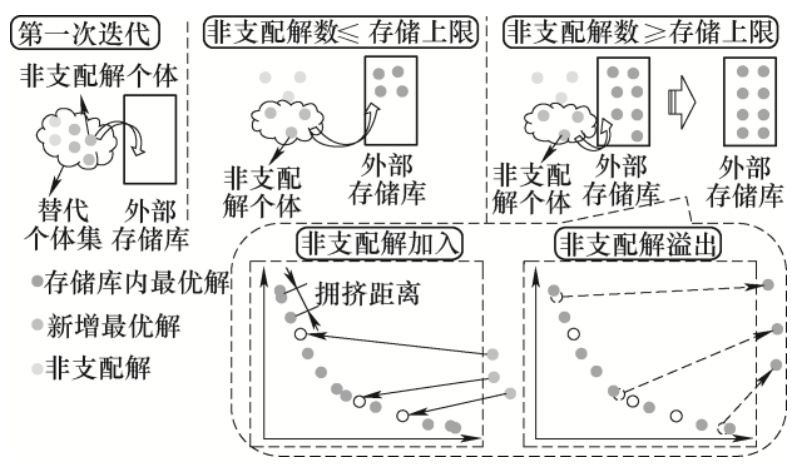

图 2 外部储存库更新原理
在第一次迭代中, 外部储存库是一个空集。在 生成的多个备选个体中, 选择非支配解个体进入外 部储存库。当储存库中的个体小于规定的数量时, 比外部储存库中的个体更好的替代个体将继续进入 外部储存库。当外部储存库中的个体数超过规定的 上限时, 符合条件的个体将继续进入外部储存库。 通过分析计算外部储存库中个体之间的拥挤度, 并 将高拥挤度的个体逐一消除, 直到个体数等于外部 储存库的容量。

\section{2 交叉操作}

交叉操作是模拟生物在基因遗传过程中通过基 因重组的形式组成后代。这里采用数字的编码方式表 达产品的信息。假设 A、B、C 三款产品的加工数量 分别为 $4,3,3$; 产品总数为 10 。随机生成一组可行 编码: 1-3-7-5-2-6-4-9-8-10, 那么上述这一组可行的 编码所代表的排序方案为 A-A-B-B-A-B-A-C-C-C。

交叉操作方法如图 3 所示。首先, 随机选择 2 个父代个体, 同时随机生成 2 个交叉点将上述个体 分割为 3 段。其次, 搜索父代 1 中间部分的序号所 对应在父代 2 中的序列, 以此替换父代 1 的中间部 分。对父代 2 执行相同的操作。最后, 形成 2 个新 的子代个体。

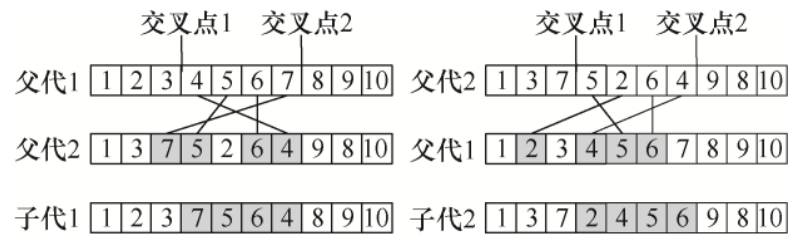

图 3 交叉操作

\section{3 变异操作}

变异操作则是模拟生物在遗传进化中的变异动 作。自然界的生物历经长期自然选择和种群繁衍使 优秀基因持续累积, 劣质基因逐次淘汰。这种进化 机制使种群基因变更逐渐沿固定方向缓慢进化。变 异操作会形成新的物种性状, 从而挖掘群体中个体 多样性。

变异操作能够增强算法局部的随机搜索能力。 一般而言, 为避免破坏已有的接近最优解的值, 变 异概率的取值较小。如图 4 所示, 通过随机变异的 方法进行变异点的选择, 并将个体在变异点前后的 基因互换，生成变异后的个体。

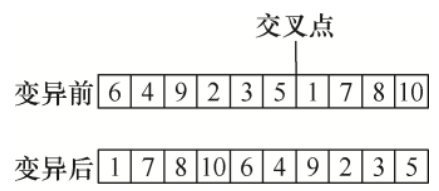

图 4 变异操作 
自适应交叉和变异概率的计算公式如下 ${ }^{[28]}$

$$
\begin{gathered}
P_{c}= \begin{cases}P_{c 1}-\frac{P_{c 2}\left(f^{\prime}-f_{\text {avg }}\right)}{f_{\max }-f_{\text {avg }}} & f^{\prime} \geqslant f_{\text {avg }} \\
P_{c 1} & f^{\prime}<f_{\text {avg }}\end{cases} \\
P_{m}= \begin{cases}P_{m 1}-\frac{P_{m 2}\left(f_{\max }-f\right)}{f_{\max }-f_{\text {avg }}} & f \geqslant f_{\text {avg }} \\
P_{m 1} & f<f_{\text {avg }}\end{cases}
\end{gathered}
$$

式中， $f_{\text {max }}$ 表示种群中适应度最大值； $f_{\text {avg }}$ 表示每 代种群中适应度平均值; $f^{\prime}$ 则为两个交叉个体中具 有较大适应度的个体所对应的值; $f$ 表示变异个体 适应度值; $P_{c 1}$ 表示初始交叉概率; $P_{c 2}$ 表示交叉概 率调整系数; $P_{m 1}$ 表示初始变异概率; $P_{m 2}$ 表示变异 概率调整系数。
双层交互式遗传算法具体步骤如下(图 5)。

(1) 在数据库中提取待分解的总订单信息, 若总 订单量不大于企业日生产量, 将全部订单进行分解。 将总订单设置为订单分解个体 Tpop, 并转至步骤(4), 若总订单量大于企业日生产量, 则进入步骤(2)。

（2）基于订单的紧急度得到临界订单信息，并 储存在临界订单空间。

（3）分析临界订单，若临界订单量大于企业日 生产量, 那么将全部临界订单进行分解, 并设置为 订单分解个体 Tpop 之后进入步骤(4); 若临界订单量 不大于企业日生产量, 将临界订单为订单分解个体 Tpop, 直接进入步骤(4)。

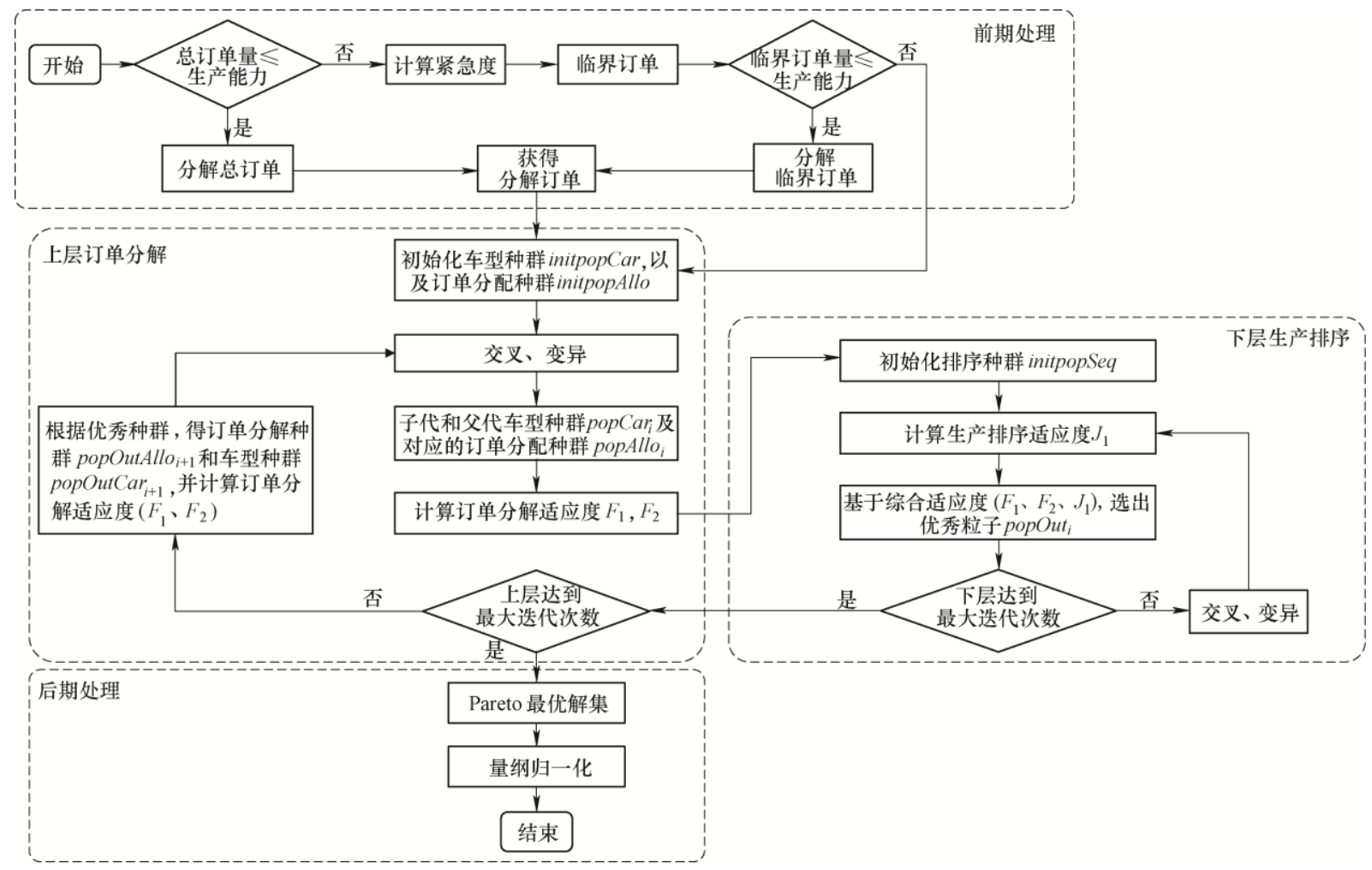

图 5 双层交互式遗传算法流程

(4) 随机产生初始化车型种群 initpopCar, 并 获得相应的订单分解种群 initpopAllo。值得注意的 是, 为了保证同种车型订单同一时间分解, 订单分 解的迭代优化遗传依赖于车型种群及其对应的订单 分解种群 popAllo ${ }_{i}$, 而与排序问题相互影响迭代的 只是订单分解种群。

(5) 对车型种群进行交叉、变异, 获得新的车

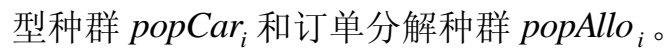

(6) 计算其订单分解的适应度 $\left(F_{1}, F_{2}\right)$ 。

(7) 根据步骤(5)的订单分解个体 popAllo ${ }_{i}$ 或者订 单分解个体 Tpop, 随机生成初始化排序种群 initpopSeq。
(8) 计算投产排序的适应度 $J_{1}$ 。

(9) 根据订单分解和投产排序的适应度 $\left(F_{1}, F_{2}, J_{1}\right)$, 应用小生镜技术, 得到 Pareto 前沿解, 按照前述个体择优的方式选出优秀个体 popOut ${ }_{i}$ 。

(10) 判断投产排序操作是否达到最大迭代次 数, 若未达到, 进入步骤(11), 否则进入步骤(12)。

(11) 将步骤(9)中的优秀个体进行交叉、变异, 获得新的排序种群 $p o p_{i+1}$, 转至步骤(8)。

(12) 判断上层订单分解的最大迭代次数, 若订 单分解没有达到最大迭代次数, 则进入步骤(13), 否则进入步骤(14)。

(13) 根据步骤(9)得到的优秀个体, 得到订单分 
解种群 popOutAllo ${ }_{i+1}$ 和车型种群 popOutCar $r_{i+1}$ 信息, 即此处得到的车型种群为综合性能较好的订单分解 优秀个体, 下一步进入步骤(5)的交叉变异操作。

(14) 得到主从优化目标函数 $F_{1}, F_{2}, J_{1}$ 最终的

Pareto 非支配前沿解集。

（15）结合实际情况, 对非支配解进行量纲归一 化操作得到最终的订单分解和排序结果。

(16) 结束。

\section{3 案例研究}

本节以某车企的 3 条并行混装线为例, 分析其 一周内的企业实际订单分解情况, 并与多阶段遗传
算法以及本文所提的双层交互式遗传算法进行对 比，验证所提方法和算法的有效性。根据企业当 前总订单可知, 需要加工的车型有 21 款, 其生产 数量、与各混装线的匹配度、交期时间等信息如 表 3 所示。目前企业有三条并行混装线的日生产 能力均为 8 辆。当前订单计划时间为 2019 年 1 月 2 日。

三条并行混装线的工作站均为 15 个。 21 款产 品在各个站点的具体作业时间如表 4 所示。值得注 意的是, 表 4 所示各产品在各个站点的作业时间均 为假设产品与该混装线完全匹配下的作业时间。如 果产品被分配至其他产线, 则根据表 4 的匹配度对 其作业时间进行相应的调整。

表 3 车型订单信息

\begin{tabular}{|c|c|c|c|c|c|c|c|c|c|c|c|}
\hline \multirow{2}{*}{ 车型编号 } & \multirow{2}{*}{ 生产数量 } & \multicolumn{3}{|c|}{ 匹配度 } & \multirow{2}{*}{ 交期时间 } & \multirow{2}{*}{ 车型编号 } & \multirow{2}{*}{ 生产数量 } & \multicolumn{3}{|c|}{ 匹配度 } & \multirow{2}{*}{ 交期时间 } \\
\hline & & $\mathrm{A}$ 线 & $\mathrm{B}$ 线 & $\mathrm{C}$ 线 & & & & $\mathrm{A}$ 线 & $\mathrm{B}$ 线 & $\mathrm{C}$ 线 & \\
\hline 1 & 5 & 1 & 0.8 & 0.96 & 2019.01.24 & 12 & 5 & 0.8 & 0.85 & 1 & 2019.02 .12 \\
\hline 2 & 4 & 0.8 & 1 & 0.92 & 2019.01 .25 & 13 & 8 & 0.85 & 0.93 & 1 & 2019.02 .13 \\
\hline 3 & 3 & 1 & 0.89 & 0.97 & 2019.01.25 & 14 & 10 & 1 & 0.95 & 0.89 & 2019.02 .14 \\
\hline 4 & 4 & 0.89 & 0.95 & 1 & 2019.01.27 & 15 & 4 & 0.82 & 0.87 & 1 & 2019.02 .15 \\
\hline 5 & 6 & 0.82 & 1 & 0.92 & 2019.01.28 & 16 & 6 & 0.89 & 1 & 0.97 & 2019.02 .15 \\
\hline 6 & 4 & 1 & 0.81 & 0.72 & 2019.02 .01 & 17 & 7 & 1 & 0.94 & 0.88 & 2019.02 .16 \\
\hline 7 & 2 & 0.97 & 0.82 & 1 & 2019.02.04 & 18 & 3 & 0.88 & 0.87 & 1 & 2019.02 .17 \\
\hline 8 & 6 & 0.85 & 1 & 0.96 & 2019.02.06 & 19 & 4 & 0.89 & 1 & 0.89 & 2019.02 .18 \\
\hline 9 & 1 & 0.89 & 1 & 0.79 & 2019.02.07 & 20 & 6 & 1 & 0.92 & 0.92 & 2019.02 .18 \\
\hline 10 & 2 & 0.84 & 0.85 & 1 & 2019.02.09 & 21 & 4 & 0.96 & 1 & 0.89 & 2019.02 .19 \\
\hline 11 & 6 & 1 & 0.89 & 0.94 & 2019.02.10 & & & & & & \\
\hline
\end{tabular}

表 4 各产品的工位时间

\begin{tabular}{|c|c|c|c|c|c|c|c|c|c|c|c|c|c|c|c|}
\hline $\begin{array}{l}\text { 产品 } \\
\text { 编号 }\end{array}$ & 站 1 & 站 2 & 站 3 & 站 4 & 站 5 & 站 6 & 站 7 & 站 8 & 站 9 & 站 10 & 站 11 & 站 12 & 站 13 & 站 14 & 站 15 \\
\hline 1 & 36.57 & 29.48 & 44.24 & 33.57 & 31.77 & 51.96 & 24.93 & 32.94 & 35.58 & 36.48 & 30.69 & 30.33 & 40.92 & 24.43 & 32.17 \\
\hline 2 & 28.08 & 28.95 & 43.20 & 34.41 & 33.60 & 45.76 & 21.85 & 34.47 & 37.14 & 44.24 & 38.13 & 26.58 & 29.01 & 22.50 & 25.28 \\
\hline 3 & 33.30 & 28.88 & 48.76 & 38.58 & 35.55 & 45.36 & 25.28 & 31.74 & 35.43 & 42.16 & 27.30 & 33.99 & 32.97 & 22.90 & 28.10 \\
\hline 4 & 28.17 & 28.88 & 45.28 & 38.82 & 33.03 & 53.24 & 30.85 & 30.54 & 36.03 & 48.28 & 29.25 & 30.24 & 32.64 & 32.10 & 32.45 \\
\hline 5 & 30.51 & 25.30 & 41.12 & 37.44 & 36.69 & 48.56 & 29.45 & 30.21 & 37.59 & 47.68 & 38.85 & 35.82 & 36.51 & 29.05 & 26.92 \\
\hline 6 & 29.58 & 24.73 & 45.32 & 34.35 & 35.61 & 42.20 & 22.8 & 34.56 & 33.75 & 51.80 & 32.22 & 34.20 & 37.98 & 31.75 & 25.35 \\
\hline 7 & 34.26 & 26.55 & 41.64 & 35.19 & 35.97 & 50.48 & 29.45 & 30.93 & 35.61 & 40.64 & 35.16 & 30.81 & 36.00 & 33.23 & 27.95 \\
\hline 8 & 36.06 & 28.65 & 43.40 & 36.36 & 37.11 & 38.16 & 26.75 & 35.58 & 31.47 & 46.44 & 27.51 & 30.84 & 31.56 & 23.67 & 23.48 \\
\hline 9 & 32.97 & 24.13 & 45.28 & 38.19 & 32.07 & 53.32 & 28.10 & 33.81 & 34.17 & 53.84 & 26.49 & 37.26 & 38.91 & 22.28 & 23.43 \\
\hline 10 & 30.66 & 29.50 & 45.88 & 38.88 & 34.89 & 49.88 & 24.08 & 31.89 & 36.57 & 38.00 & 27.93 & 35.40 & 39.75 & 27.18 & 24.35 \\
\hline 11 & 31.26 & 30.70 & 43.76 & 36.99 & 29.70 & 53.44 & 27.53 & 34.14 & 36.39 & 47.08 & 35.58 & 32.97 & 33.18 & 33.40 & 31.55 \\
\hline 12 & 28.05 & 24.93 & 46.32 & 30.99 & 32.76 & 43.32 & 30.10 & 32.22 & 37.77 & 49.96 & 30.24 & 36.84 & 33.03 & 22.60 & 29.83 \\
\hline 13 & 32.22 & 29.55 & 42.24 & 38.22 & 31.83 & 42.12 & 21.90 & 32.46 & 36.90 & 47.36 & 32.10 & 36.03 & 38.52 & 33.13 & 32.32 \\
\hline 14 & 26.97 & 25.40 & 39.28 & 30.09 & 34.65 & 53.92 & 22.73 & 31.68 & 34.29 & 50.32 & 33.99 & 33.93 & 40.77 & 23.48 & 30.93 \\
\hline 15 & 34.05 & 28.40 & 47.32 & 36.36 & 31.38 & 45.76 & 23.78 & 32.91 & 34.23 & 40.68 & 30.00 & 37.50 & 36.93 & 25.32 & 23.40 \\
\hline 16 & 36.36 & 26.42 & 47.24 & 38.91 & 26.79 & 45.56 & 27.45 & 33.96 & 35.85 & 47.04 & 38.16 & 26.58 & 28.74 & 29.80 & 28.78 \\
\hline 17 & 31.92 & 28.58 & 48.68 & 35.58 & 32.70 & 45.28 & 21.83 & 35.25 & 36.87 & 41.72 & 37.47 & 26.28 & 35.19 & 22.23 & 27.98 \\
\hline 18 & 27.66 & 27.08 & 38.48 & 30.75 & 34.71 & 47.92 & 29.40 & 33.81 & 35.79 & 42.24 & 38.10 & 36.93 & 31.38 & 27.13 & 23.93 \\
\hline 19 & 26.70 & 28.00 & 41.76 & 37.23 & 35.70 & 42.96 & 28.08 & 31.50 & 35.28 & 45.64 & 34.23 & 37.05 & 39.27 & 24.75 & 32.30 \\
\hline 20 & 30.30 & 28.58 & 47.32 & 35.13 & 34.95 & 40.36 & 28.83 & 30.27 & 30.90 & 39.00 & 27.33 & 28.17 & 29.31 & 30.83 & 31.03 \\
\hline 21 & 35.52 & 29.78 & 42.80 & 36.63 & 28.32 & 38.08 & 21.98 & 34.53 & 29.97 & 52.48 & 29.85 & 35.52 & 34.44 & 28.85 & 27.45 \\
\hline
\end{tabular}


其具体调整方式如下: $m$ 型产品在站点 $s$ 上的 作业时间为 $t_{s}^{(m)}$, 当产品分解至生产线 $l$ 时, 它在站 点 $s$ 上的作业时间为 $t_{s}^{(m)} / \sqrt{M A_{m l}}$; 其中, $M A_{m l}$ 表示 $m$ 型产品与混装线 $l$ 之间的匹配度。

利用双层交互式遗传算法所得到 Pareto 前沿解 如表 5 所示, 所得前沿解数量为 66 个。由于 Pareto 前沿解是一个最优解集, 当其数量较多时, 对排程 人员的决策造成困扰。如图 6 中双层交互式遗传算 法流程所述, 在 Pareto 前沿解的基础上, 依据实际 生产过程中企业对个目标的重视程度及经验分析, 采用量纲归一化方法进行最终决策。通过减少初始 赋权所带来的主观性, 为企业的最终决策提供数学 依据。在量纲归一化之前, 需对各个子目标采用均 值化方法进行无量纲化处理 ${ }^{[29]}$, 均值化方法如下

$$
y_{i j}=\frac{x_{i j}}{\Delta x_{j}}=\frac{\omega_{j}\left(x_{i j}-x_{j \min }\right)}{x_{j \max }-x_{j \min }}
$$

式中, $x_{j \max } 、 x_{j \min }$ 为 Pareto 前沿解中同一个目标
函数 $j$ 所对应的两个极值; $x_{i j}$ 是归一化前的值; $\omega_{j}$ 为目标 $j$ 的需求占比权重。由于产线工作负荷 $J_{1}$ 的 性能是越小越好, 而紧急度 $F_{1}$ 和匹配度 $F_{2}$ 值越大性 能越好, 故基于订单分解和投产排序的量纲归一化 适应度函数值为

$$
\begin{gathered}
F_{n}=\max \left[\frac{\omega_{1}\left(J_{1}-J_{1 \max }\right)}{J_{1 \min }-J_{1 \max }}+\frac{\omega_{1}\left(F_{1}-F_{1 \max }\right)}{F_{1 \max }-F_{1 \min }}+\right. \\
\left.\frac{\omega_{3}\left(F_{2}-F_{2 \max }\right)}{F_{2 \max }-F_{2 \min }}\right]
\end{gathered}
$$

根据企业实际需求取工作负荷 $J_{1}$ 、紧急度 $F_{1}$ 和 匹配度 $F_{2}$ 的权重分别为 $\omega_{1}=0.2, \omega_{2}=0.45$, $\omega_{3}=0.35$ 。最终得到订单分解与生产排序的 Pareto 前沿解集的综合性能排序情况如表 5 所示。上述各 目标的权重会根据定制化需求以及企业实际需求的 变动而改变。运行环境为 Matlab 2018b, 求解上述 多目标决策模型。种群大小为 100 , 初始交叉概率 0.8 , 交叉概率调整系数 0.3 , 变异概率设为 0.1 , 变

\begin{tabular}{|c|c|c|c|c|c|c|c|c|}
\hline 解集编号 & 综合值 & 紧急度 $F_{1}$ & 匹配度 $F_{2}$ & 工作负荷 $J_{1}$ & $\mathrm{~A}$ 线 & $\mathrm{B}$ 线 & $\mathrm{C}$ 线 & 天数 \\
\hline \multirow{5}{*}{1} & \multirow{5}{*}{0.8} & \multirow{5}{*}{85.34} & \multirow{5}{*}{114.1} & \multirow{5}{*}{23435.07} & $14,14,14,14,14,14,14,14$ & $14,17,14,17,17,17,17,17$ & $12,12,12,12,12,17,12,12$ & 第 1 天 \\
\hline & & & & & $21,21,21,19,21,21,21,21,21$ & $12,5,19,12,12,12,12,9$ & $5,5,5,5,5,3,3,3$ & 第 2 天 \\
\hline & & & & & $19,19,19,19,19,19,19,19$ & $19,19,16,16,16,16,16,16$ & $13,13,13,13,13,13,13,13$ & 第 3 天 \\
\hline & & & & & $18,18,20,20,20,20,20,20$ & $18,18,15,15,15,15,18,4$ & $11,7,7,11,11,4,4,4$ & 第 4 天 \\
\hline & & & & & $11,6,11,11,6,10,6,10$ & $6,1,1,1,2,2,2,2$ & $8,8,8,1,8,8,1,8$ & 第 5 天 \\
\hline \multirow[t]{3}{*}{$\cdots$} & \multirow[t]{3}{*}{$\cdots$} & \multirow[t]{3}{*}{$\cdots$} & \multirow[t]{3}{*}{$\cdots$} & \multirow[t]{3}{*}{$\cdots$} & $\cdots$ & $\cdots$ & $\cdots$ & $\cdots$ \\
\hline & & & & & $6,6,5,5,5,5,5,5$ & $2,2,2,2,1,1,1,1$ & $4,1,3,3,3,4,4,4$ & 第 1 天 \\
\hline & & & & & $11,11,12,11,12,12,12,12$ & $8,8,8,11,11,10,10,11$ & $7,8,7,8,8,6,9,6$ & 第 2 天 \\
\hline \multirow[t]{3}{*}{34} & \multirow[t]{3}{*}{0.46} & \multirow[t]{3}{*}{79.88} & \multirow[t]{3}{*}{113.11} & \multirow[t]{3}{*}{26825.74} & $14,14,14,14,14,14,14,14$ & $14,13,13,13,13,13,13,13$ & $12,13,12,12,12,12,12,12$ & 第 3 天 \\
\hline & & & & & $18,18,15,15,19,18,18,18$ & $14,15,15,17,17,17,17,17$ & $16,16,16,16,16,16,17,17$ & 第 4 天 \\
\hline & & & & & $20,21,20,21,20,20,20,20$ & $21,21,21,21,21,19,19,19$ & $19,19,19,19,19,19,19,19$ & 第 5 天 \\
\hline \multirow[t]{3}{*}{$\cdots$} & \multirow[t]{3}{*}{$\cdots$} & \multirow[t]{3}{*}{$\cdots$} & \multirow[t]{3}{*}{$\cdots$} & \multirow[t]{3}{*}{$\cdots$} & $\cdots$ & $\cdots$ & $\cdots$ & $\cdots$ \\
\hline & & & & & $21,21,17,21,17,21,17,17$ & $13,13,17,13,13,9,17,17$ & $13,13,13,13,2,2,2,2$ & 第 1 天 \\
\hline & & & & & $11,21,11,11,21,11,11,21$ & $6,6,1,6,10,6,11,10$ & $1,8,1,8,8,1,8,1$ & 第 2 天 \\
\hline \multirow[t]{3}{*}{66} & \multirow[t]{3}{*}{0.33} & \multirow[t]{3}{*}{78.67} & \multirow[t]{3}{*}{113.12} & \multirow[t]{3}{*}{26716.50} & $19,19,19,19,19,19,19,19$ & $14,19,19,19,19,14,14,14$ & $14,8,7,8,14,14,14,7$ & 第 3 天 \\
\hline & & & & & $18,16,16,18,16,18,18,18$ & $5,16,16,5,5,5,5,16$ & $14,12,5,12,12,12,14,12$ & 第 4 天 \\
\hline & & & & & $12,12,20,20,20,20,20,20$ & $12,12,4,12,4,12,12,4$ & $4,3,3,3,15,15,15,15$ & 第 5 天 \\
\hline
\end{tabular}
异概率调整系数为 0.08 , 最大迭代次数 100 次。

表 5 订单分解与生产排序的 Pareto 前沿解集

企业的生产计划方案是基于人工经验和交期优 先等因素制定的。首先, 对已有订单依据车型与各 混装线之间的匹配度进行订单分解; 其次, 根据产 品交期对一个周期内的订单整体投产排序; 最后, 通过各条混装线生产能力, 订单连续性等进行调整。 依据上述原则, 得到的企业实际方案如表 6 所示。
其相应的紧急度 $F_{1}$ 为 79.04 , 匹配度 $F_{2}$ 为 119.7 , 工 作负荷 $J_{1}$ 为 $24300.66 \mathrm{~min}$ 。

基于多阶段遗传算法的方案确定方法是将 目标函数按照重要程度进行排序。首先, 对最重 要的子目标进行寻优; 其次, 在首选子目标最优 的基础上, 求解次要子目标的最优解。重复上述 
操作直至最后一个子目标, 或者某一目标再也找 不到更优解为止。本文根据企业实际需求出发, 确定各子目标的重要次序如下: $F_{1} 、 F_{2} 、 J_{1}$ 。
其优化结果如表 6 所示。其相应的紧急度 $F_{1}=86.58$, 匹配度 $F_{2}=117.36$, 工作负荷 $J_{1}=25745.33 \mathrm{~min}$ 。

表 6 订单分解与生产排序方案

\begin{tabular}{|c|c|c|c|c|c|c|c|}
\hline 方案 & 工作负荷 $J_{1}$ & 紧急度 $F_{1}$ & 匹配度 $F_{2}$ & $A$ 线 & $\mathrm{B}$ 线 & $\mathrm{C}$ 线 & 天数 \\
\hline \multirow{5}{*}{$\begin{array}{c}\text { 企业实际方 } \\
\text { 案 }\end{array}$} & \multirow{5}{*}{25745.33} & \multirow{5}{*}{79.04} & \multirow{5}{*}{119.70} & $1,1,1,1,1,3,3,3$ & $2,2,2,2,5,5,5,5$ & $4,4,4,4,7,7,10,10$ & 第 1 天 \\
\hline & & & & $6,6,6,6,11,11,11,11$ & $5,5,8,8,8,8,8,8$ & $12,12,12,12,12,12,12,12$ & 第 2 天 \\
\hline & & & & $11,11,14,14,14,14,14,14$ & $9,16,16,16,16,16,16,19$ & $12,12,12,12,13,13,13,13$ & 第 3 天 \\
\hline & & & & $14,14,14,14,17,17,17,17$ & $19,19,19,19,19,19,19,19$ & $13,13,13,13,15,15,15,15$ & 第 4 天 \\
\hline & & & & $17,17,17,20,20,20,20,20$ & $19,19,19,21,21,21,21,21$ & $18,18,18,18,18,20,21,21$ & 第 5 天 \\
\hline \multirow{5}{*}{$\begin{array}{c}\text { 多阶段遗传 } \\
\text { 算法 }\end{array}$} & \multirow{5}{*}{24300.66} & \multirow{5}{*}{86.58} & \multirow{5}{*}{117.36} & $1,1,1,1,1,3,3,3$ & $2,2,2,2,5,5,5,5$ & $4,4,4,4,5,5,6,6$ & 第 1 天 \\
\hline & & & & $6,6,11,11,11,11,11,11$ & $8,8,8,8,8,8,9,12$ & $7,7,10,10,12,12,12,12$ & 第 2 天 \\
\hline & & & & $14,14,14,14,14,14,14,14$ & $13,13,13,13,13,13,13,14$ & $12,12,12,12,12,12,12,13$ & 第 3 天 \\
\hline & & & & $14,17,17,17,17,17,17,17$ & $16,16,16,16,16,16,18,19$ & $15,15,15,15,18,18,18,18$ & 第 4 天 \\
\hline & & & & $20,20,20,20,20,19,19,19$ & $19,19,19,19,19,19,19,19$ & $19,21,21,21,21,21,21,21$ & 第 5 天 \\
\hline \multirow{5}{*}{$\begin{array}{c}\text { 交互式遗传 } \\
\text { 算法 }\end{array}$} & \multirow{5}{*}{23435.07} & \multirow{5}{*}{85.34} & \multirow{5}{*}{114.1} & $14,14,14,14,14,14,14,14$ & $14,17,14,17,17,17,17,17$ & $12,12,12,12,12,17,12,12$ & 第 1 天 \\
\hline & & & & $21,21,21,19,21,21,21,21,21$ & $12,5,19,12,12,12,12,9$ & $5,5,5,5,5,3,3,3$ & 第 2 天 \\
\hline & & & & $19,19,19,19,19,19,19,19$ & $19,19,16,16,16,16,16,16$ & $13,13,13,13,13,13,13,13$ & 第 3 天 \\
\hline & & & & $18,18,20,20,20,20,20,20$ & $18,18,15,15,15,15,18,4$ & $11,7,7,11,11,4,4,4$ & 第 4 天 \\
\hline & & & & $11,6,11,11,6,10,6,10$ & $6,1,1,1,2,2,2,2$ & $8,8,8,1,8,8,1,8$ & 第 5 天 \\
\hline
\end{tabular}

图 6 展示了不同方法所对应的产品投产方案 下, 各条生产线与产品的匹配度变化图。从图 6 中 可以看出, 企业实际方案(图中虚线)在产品匹配度 上做到最优, 除了 $\mathrm{C}$ 线最后 3 款车型外, 其余的均 能够实现产品和生产线完全匹配(匹配度为 1 )。而采 用多阶段遗传算法(图中双点划线)和交互式遗传算 法(图中实线)则在匹配度上呈现了较大的波动性。 这是因为在企业实际方案中, 排程人员进行订单分 解时, 首先考虑的是基于匹配度的产品交期约束; 而多阶段遗传算法首先考虑的是订单紧急度, 交互 式遗传算法则是综合考虑各方因素。

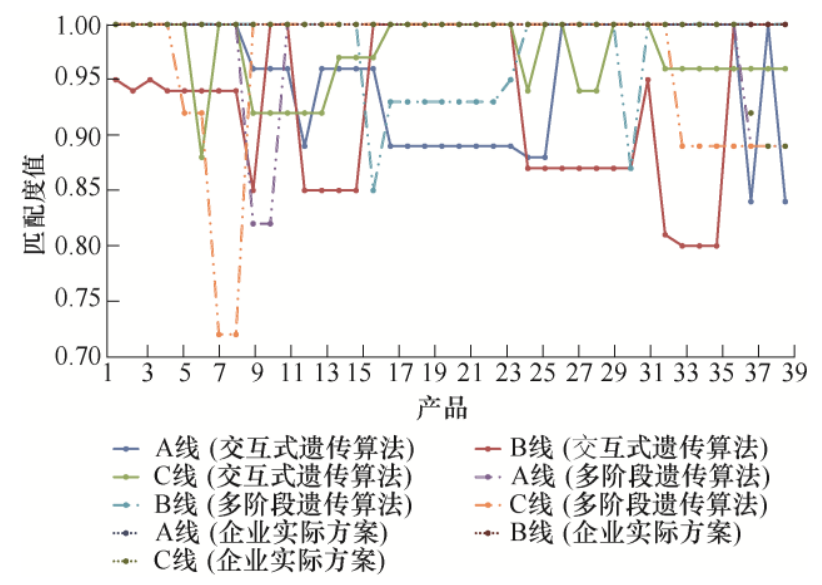

图 6 各方案对应产品与生产线匹配度变化图
虽然企业实际方案匹配度能够做到最佳, 但是 从图 7 可以看出, 它所导致的工作负荷却是最差 的。相对于交互式遗传算法的结果, 企业实际方 案和多阶段遗传算法所导致的空闲和堵塞时间分 别增加了 $2310 \mathrm{~min}$ 和 $865 \mathrm{~min}$ 。由于混装线一次 生产多种产品的特性, 这些产品在各个工作站的 作业时间存在一定的差异，这需要在投产排序时 充分地被考虑。采用主从关联优化模型, 由于同 时考虑了混装线各工作站产品作业时间的波动 性, 使得所得到的方案在堵塞和空闲上做的更好。 因此, 与两种算法对比, 在都满足交期的情况下, 减少了产能的浪费。

由于多阶段遗传算法的算法特性，其最终结 果是由前一阶段最优解结果的基础上进行优化, 这必定损失了大量的求解域。随着阶段数量的递 增, 其损失的求解域呈现指数级增长。因此, 该 方法难以达到一个综合最优解。如表 6 所示, 虽 然多阶段遗传算法所得到紧急度最大, 但是其匹 配度有所下降, 由此导致的空闲和堵塞时间也随 之增加。

另外, 考虑到企业实际需求往往瞬息万变, 根 据双层交互式遗传算法所得到的 Pareto 解集可以给 排程人员以更多的选择, 使其能够在考虑数学模型 的基础上根据企业实际需求做出更为合理的选择。 


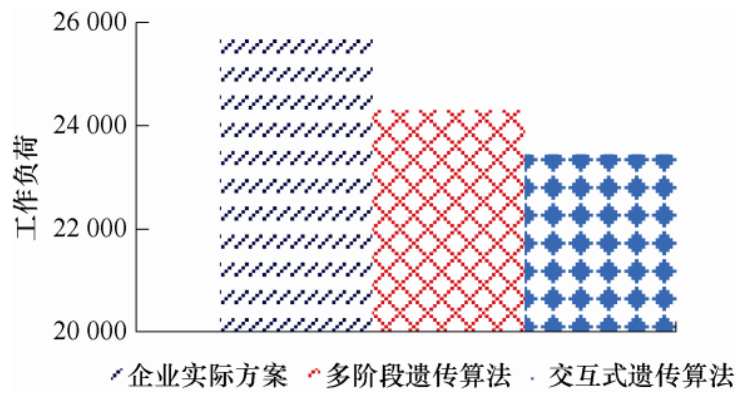

图 7 三种方案对应工作负荷

为了进一步分析遗传算法在解决这类问题的有 效性, 我们利用上述企业实际案例通过其他相关算 法与本文所提遗传算法的进行性能对比。由于模拟 退火算法在生产线规划、组合优化等问题方面也展 现良好的性能 ${ }^{[30]}$, 故选择模拟退火算法与双层交互 式遗传算法对比。通过如表 7 所示结果可以看出, 除了在紧急度方面两种算法获得几乎相同的函数 值, 遗传算法在负荷和匹配度方面均取得了比模拟 退火算法更优的解。因此, 可以认为遗传算法在求 解包含 0-1 离散变量的复杂模型相对模拟退火算法 效果更佳。

表 7 双层规划算法结果对比

\begin{tabular}{lccc}
\hline 方案 & 工作负荷 $J_{1}$ & 紧急度 $F_{1}$ & 匹配度 $F_{2}$ \\
\hline 交互式遗传算法 & 23435.07 & 85.3411 & 114.1 \\
模拟退火算法 & 23675.21 & 85.3424 & 112.95 \\
\hline
\end{tabular}

\section{4 结论}

(1) 分析了订单分解和投产排序在企业生产计 划过程中主从关联关系, 考虑企业实际多条并行混 装线之间的不等效特性, 建立以紧急度、匹配度以 及工作负荷为多目标的主从关联优化模型。

(2) 提出了一种结合 Pareto 前沿解的双层交互 式遗传算法。引入自适应调整方法对交叉概率和变 异概率进行改进, 并采用小生境技术保证种群多样 性。在 Pareto 前沿解的基础上, 利用量纲归一化方 法进行解集排序, 增强解集的可选性和适应性。

(3) 以某车企 3 条并行不等效混装线为研究对 象, 通过与多阶段遗传算法、企业实际生产计划以 及模拟退火算法进行对比, 验证了改进双层交互式 遗传算法的合理性和有效性。

\section{参 考 文 献}

[1] 张泽群, 唐敦兵, 金永乔, 等. 信息物联驱动下的离散 车间自组织生产调度技术 $[\mathrm{J}]$. 机械工程学报, 2018 , 54(16): 34-44.
ZHANG Zequn, TANG Dunbing, JIN Yongqiao, et al. Self-organizing production technology for discrete workshop scheduling driven by internet of things[J]. Journal of Mechanical Engineering, 2018, 54(16): 34-44.

[2] 谢卫红, 王永健, 成晓超. IT 能力对竞争优势的影响机 制: 以制造柔性为中介[J]. 工业工程，2012，15(2): 59-65.

XIE Weihong, WANG Yongjian, CHENG Xiaochao. Influencing mechanism analysis of it capability on competitiveness: The mediating effect of manufacturing flexibility[J]. Industrial Engineering Journal, 2012, 15(2): 59-65.

[3] HOU L, JIAO R J. Data-informed inverse design by product usage information: A review, framework and outlook[J]. Journal of Intelligent Manufacturing, 2020,

31(3): 529-552.

[4] 李启锐, 彭志平, 崔得龙. 单件小批量生产模式下 MRP 的柔性化改进 [J]. 江苏科技大学学报, 2016, 30(4): 355-361.

LI Qirui, PENG Zhiping, CUI Delong. Flexible improvement of MRP in jobbing work circumstance[J]. Journal of Jiangsu University of Science and Technology, 2016, 30(4): 355-361.

[5] 郑力, 江平宇, 乔立红, 等. 制造系统研究的挑战和前 沿[J]. 机械工程学报, 2010，46(21): 128-140.

ZHENG Li, JIANG Pingyu, QIAO Lihong, et al. Challenges and frontiers of manufacturing systems $[\mathrm{J}]$. Journal of Mechanical Engineering, 2010, 46(21): 128-140.

[6] 熊先青, 袁莹莹, 牛怡婷, 等. 大规模定制家具 ERP 系统的构建及其关键技术 $[\mathrm{J}]$. 林业工程学报，2019， 4(4): 162-168.

XIONG Xianqing, YUAN Yingying, NIU Yiting, et al. Research on key technologies of ERP system for mass customization furniture production[J]. Journal of Forestry Engineering, 2019, 4(4): 162-168.

[7] WUNSCH D , BRATUKHIN A. Multilevel order decomposition in distributed production[C/CD $] / /$ Emerging Technologies and Factory Automation, 2007. ETFA. IEEE Conference on. IEEE， 2007.

[8] 王静, 潘开灵, 刘朝, 等. 云制造平台下订单可分解的 协同生产计划模型及求解 $[\mathrm{J}]$. 上海交通大学学报, 2018, 52(12): 117-124.

WANG Jing, PAN Kailing, LIU Ao, et al. The model and solution for collaborative production planning with order 
splitting in cloud manufacturing platform[J]. Journal of Shanghai Jiao Tong University, 2018, 52(12): 117-124.

[9] 陈旻, 陆志强. 基于订单总拖期的仓储作业调度建模与 优化 $[J]$. 上海交通大学学报, 2009, 12(1): 1916-1922. CHEN Min, LU Zhiqiang. Modeling and optimization on operation scheduling with total tardiness objective for a storage system[J]. Journal of Shanghai Jiao Tong University, 2009, 12(1): 1916-1922.

[10] 郑华林, 刘飞, 熊峰, 等. 一种新型的单件小批量生产 车间调度方法[J]. 重庆大学学报, 2001, 24(1): 20-23. ZHENG Hualin, LIU Fei, XIONG Feng, et al. A study on the job-shop scheduling problem[J]. Journal of Chongqing University, 2001, 24(1): 20-23.

[11] 张洁, 张朋, 刘国宝. 基于两阶段蚁群算法的带非等效 并行机的作业车间调度 [J]. 机械工程学报, 2013, 49(6): 140-148.

ZHANG Jie, ZHANG Peng, LIU Guobao. Two-stage ant colony algorithm based job shop scheduling with unrelated parallel machines[J]. Journal of Mechanical Engineering, 2013, 49(6): 140-148.

[12] DAR-EL E , CUCUY S. Optimal mixed-model sequencing for balanced assembly lines[J]. Omega, 1977, 5(3): $333-342$.

[13] 范林胜, 邓建新, 陈一辉, 等. 基于仿真的某发动机混 合生产线的均衡改进 $[\mathrm{J}]$. 组合机床与自动化加工技术, 2016(8): 118-123.

FAN Linsheng, DENG Jianxin, CHEN Yihui, et al. Based on simulation in a balanced improvement of engine hybrid production line[J]. Modular Machine Tool \& Automatic Manufacturing Technique, 2016(8): 118-123.

[14] FLIEDNER M, BOYSEN N. Solving the car sequencing problem via Branch \& Bound[J]. European Journal of Operational Research， 2008， 191(3): 1023-1042.

[15] 甘雅文, 侯亮, 徐昌华, 等. 考虑产品切换的客车混流 装配线排序问题 [J]. 计算机集成制造系统，2019， 25(7): 1685-1694.

GAN Yawen, HOU Liang, XU Changhua, et al. Sequencing for bus mixed-model assembly line with consideration of product switching[J]. Computer Integrated Manufacturing Systems, 2019, 25(7) : 1685-1694.

[16] 谢志强, 常宁宁, 杨静. 基于交货期紧迫度的综合调度 算法[J]. 机械工程学报，2011，47(22)：181-190.

XIE Zhiqiang, CHANG Ningning, YANG Jing. Integrated scheduling algorithm based on delivery dates urgency degree[J]. Journal of Mechanical Engineering, 2011, 47(22): 181-190.

[17] 王艳红, 于宁, 蔡明, 等. 动态制造系统生产计划与调 度协同优化 [J]. 中国机械工程, 2018, 29(22): 125-129. WANG Yanhong, YU Ning, CAI Ming, et al. Collaborative optimization of dynamic manufacturing production planning and scheduling[J]. China Mechanical Engineering, 2018, 29(22): 125-129.

[18] CHANG P T, LIN K P. Ant colony optimization system for a multi-quantitative and qualitative objective job-shop parallel machine scheduling problem[J]. International Journal of Production Research, 2009 , 46(20) : 5719-5759.

[19] AZIZOGLU M. Scheduling jobs on unrelated parallel machines to minimize regular total cost functions[J]. IIE Transactions, 2002, 31(2): 153-159.

[20] 戴隆州, 吴永明, 李少波, 等. 多目标粒子群算法在混 装线再平衡中的应用 $[\mathrm{J}]$. 计算机应用研究, 2018 , 35(1): $145-149$.

DAI Longzhou, WU Yongming, LI Shaobo, et al. Multi-objective particle swarm algorithm in mixed-model assembly line rebalancing[J]. Application Research of Computers, 2018, 35(1): 145-149.

[21] HUANG M, GUO Q, LIU J, et al. Mixed model assembly line scheduling approach to order picking problem in online supermarkets[J]. Sustainability, 2018, 10(11): 1-16.

[22] KUCUKKOC I, ZHANG D Z. Mixed-model parallel two-sided assembly line balancing problem: A flexible agent-based ant colony optimization approach[J]. Computers \& Industrial Engineering, 2016, 97(16): 58-72.

[23] ZHOU F, JIAO R J, LEI B. Bilevel game-theoretic optimization for product adoption maximization incorporating social network effects[J]. IEEE Transactions on Systems, Man, and Cybernetics: Systems, 2015, 46(8): 1047-1060

[24] 刘炜琪, 刘琼, 张超勇, 等. 基于混合粒子群算法求解 多目标混流装配线排序 [J]. 计算机集成制造系统, 2011, 17(12): 2590-2598.

LIU Weiqi, LIU Qiong, ZHANG Chaoyong, et al. Hybrid particle swarm optimization for multi-objective sequencing problem in mixed model assembly lines[J]. Computer Intergrated Manufacturing Systems, 2011, 17(12): 2590-2598.

[25] JOAQUÍN B, CANO A. Solving mixed model sequencing problem in assembly lines with serial workstations with 
work overload minimisation and interruption rules[J]. European Journal of Operational Research, 2011, 210(3): 495-513.

[26] AITZAI A, BENMEDJDOUB B, BOUDHAR M. A branch and bound and parallel genetic algorithm for the job shop scheduling problem with blocking[J]. International Journal of Operational Research, 2012, 14(3): 343-365.

[27] 郑永前, 王永生, 于胜男. 实时负荷条件下混流装配线 平衡方法研究 [J]. 现代制造工程, 2010(12)：66-72. ZHENG Yongqian, WANG Yongsheng, YU Shengnan. Mixed-model assembly line balancing based on realtime load [J]. Modern Manufacturing Engineering, 2010(12): 66-72.

[28] 姜静, 谭博学, 姜琳. 基于改进自适应遗传算法的仿真 研究[J]. 山东理工大学学报, 2008, 22(6): 10-13.

JIANG Jing, TAN Boxue, JIANG Lin. Simulation analysis based on improved self-adaptive genetic
algorithm[J]. Journal of Shandong University of Technology, 2008, 22(6): 10-13.

[29] 李玲玉, 郭亚军, 易平涛. 无量纲化方法的选取原则 [J]. 系统管理学报，2016，25(6): 1040-1045.

LI Lingyu, GUO Yajun, YI Pingtao. Analyzing the principles for choosing dimensionless methods[J]. Journal of Systems \& Management, 2016, 25(6): 1040-1045.

[30] 王炳刚. 混流加工/装配系统集成优化研究[J]. 机械工 程学报, 2010, 46(17): 118-126.

WANG Binggang. Research on integrated optimization for mixed-model fabrication/assembly systems[J]. Journal of Mechanical Engineering, 2010，46(17): 118-126.

作者简介: 张炜, 男, 1982 年出生, 博士研究生。主要研究方向为大批 量定制设计、生产线规划。

E-mail: zhangw@stu.xmu.edu.cn

侯亮(通信作者), 男, 1974 年出生, 博士，教授，博士研究生导师。主 要研究方向为产品大批量定制技术、振动噪声控制和工业大数据。

E-mail: hliang@xmu.edu.cn 